\title{
Doege-Potter Syndrome: \\ A Presumptive Case of Metastatic Hemangiopericytoma with Persistent Hypoglycemia in a 27-Year-Old Male
}

\author{
Von Lovel Zarra, ${ }^{1}$ Kenny Jun Demegillo, ${ }^{2}$ May Uyking-Naranjo, ${ }^{3}$ Ahmad Domado ${ }^{1}$ \\ ${ }^{1}$ Department of Internal Medicine, Southern Philippines Medical Center, Davao City, Philippines \\ ${ }^{2}$ Section of Oncology, Department of Internal Medicine, Southern Philippines Medical Center, Davao City, Philippines \\ ${ }^{3}$ Section of Endocrinology, Department of Internal Medicine, Southern Philippines Medical Center, Davao City, Philippines
}

\begin{abstract}
Doege-Potter syndrome (DPS) is a rare paraneoplastic condition characterized by hypoinsulinemic hypoglycemia from a solitary fibrous tumor. The underlying mechanism is the secretion of a prohormone form of insulin-like growth factor II (IGF-II) by the tumor, which causes decreased release of glucose into the circulation. We report the case of a 27-yearold Filipino male with presumptive DPS from a recurrent right temporo-zygomatic hemangiopericytoma (HPC). The complexity of DPS requires a multidisciplinary approach. Early screening for metastases from HPC may prevent the undesirable sequelae of the disease process.
\end{abstract}

Key words: Doege-Potter syndrome, hemangiopericytoma, hypoglycemia

\section{INTRODUCTION}

Hypoglycemia is a medical emergency commonly caused by medications, alcohol and sepsis. Occasionally, it can be an initial manifestation of a neoplasm, particularly islet cell tumors, such as an insulinoma. In rare instances, it can also be caused by non-islet cell entities such as solitary fibrous tumors (SFT). This condition is called Doege-Potter syndrome (DPS), which accounts for less than $5 \%$ of cases. ${ }^{1}$

DPS is a rare paraneoplastic condition characterized by hypoinsulinemic hypoglycemia secondary to ectopic secretion of the prohormone of IGF-II. ${ }^{2}$ Only 45 cases of Doege-Potter syndrome have been reported worldwide. Based on an extensive literature search, there are no documented or published cases of DPS from the Philippines. $^{3}$

\section{CASE}

A 27-year-old Filipino male presented with a painless, nodular mass on the right temporo-zygomatic area starting 10 years prior to admission. In the ensuing years, he underwent five surgical resection procedures due to recurrences on the same site. Final histopathologic diagnosis revealed hemangiopericytoma (HPC). The patient subsequently underwent radiotherapy and had no recurrence for two years.

He was apparently well until three months before admission when he began to experience malaise. Hospital admission and work-up revealed hypoglycemia, and he was discharged after resolution of the symptom. After a month, he was brought to the emergency room due to generalized tonic-clonic seizure episodes. Further workup showed hypoglycemia, prompting the consideration of an islet versus non-islet cell tumor etiology. He was discharged and was advised oncologic work-up on an outpatient basis. On the day of admission, he had another seizure episode with deterioration of sensorium.

The patient was not known to have hypertension or diabetes. He had no intake of any glucose-lowering or herbal medications. He did not smoke cigarettes, consume alcohol or use illicit drugs. Family history was unremarkable.

On initial examination, he was drowsy but remained oriented to time, place and person. He was tachycardic (108 beats per minute) and normotensive $(110 / 60 \mathrm{~mm} \mathrm{Hg})$, with a respiratory rate of 20 cycles per minute and temperature of $36.1^{\circ} \mathrm{C}$. He was also obese, with a body mass index of 30.8 $\mathrm{kg} / \mathrm{m}^{2}$. Pertinent physical findings included a depressed right temporo-zygomatic area and hepatomegaly, with a liver span of approximately $15 \mathrm{~cm}$ at the midclavicular line upon palpation. He did not have caput medusae, spider angiomata or abdominal tenderness. The other physical and neurologic examination findings were unremarkable. The initial consideration was hypoglycemia secondary to a non-islet cell tumor versus insulinoma. The patient then underwent a series of procedures where informed consent was obtained after risks and benefits were thoroughly explained with appropriate review.

\section{RESULTS}

A 72-hour fasting protocol was performed to rule out insulinoma. He experienced neuroglycopenic symptoms on the sixth hour, with a capillary blood glucose (CBG) 
of $42 \mathrm{mg} / \mathrm{dL}$. The fasting procedure was then terminated (Figure 1). Insulin and C-peptide levels were decreased, further excluding the possibility of an insulinoma or an endogenous pancreatic source (Table 1). Despite the unavailability of bioassays for IGF-II, the diagnostic algorithm focused on the clinical course of the tumor that caused hypoglycemia. This became helpful in making a presumptive diagnosis.

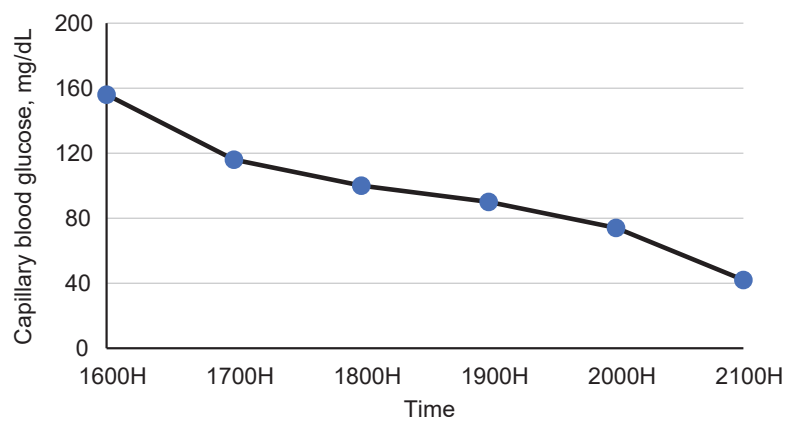

Figure 1. Capillary blood glucose monitoring during the 72-hour fast protocol. Symptomatic hypoglycemia became evident at the sixth hour.

\begin{tabular}{llll}
\multicolumn{5}{l}{ Table 1. Summary of 72-hour fasting protocol } \\
\hline Parameter & Reference range & Pre-fasting & Post-fasting \\
\hline Glucose, $\mathrm{mmol} / \mathrm{L}$ & $4.10-6.60$ & 6.5 (Normal) & 1.93 (Low) \\
Insulin, pmol/L & $2.6-24.9$ & 35.5 (High) & $<0.2($ Low) \\
C-peptide, $\mathrm{ng} / \mathrm{mL}$ & $0.78-5.19$ & 1.19 (Normal) & $3.0($ Low) \\
\hline
\end{tabular}

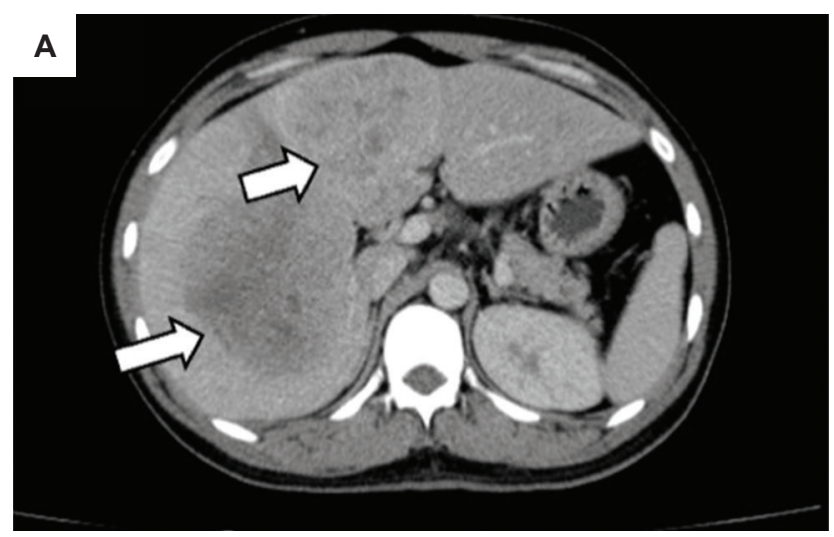

Cranial computerized tomography (CT) scan showed no evidence of tumor recurrence. However, hepatic and right adrenal metastases without pancreatic involvement were seen on abdominal CT scan. These were described as multiple, well-defined, heterogeneously enhancing ovoid lesions of different sizes predominantly on the right hepatic lobe (Figure 2A). The largest mass was visualized at segment 5 measuring $10.2 \mathrm{~cm} \times 9.1 \mathrm{~cm} \times 10.7$ $\mathrm{cm}$. In consideration of the low sensitivity (70 to $90 \%$ for all tumor sizes and less than $50 \%$ for tumor sizes 1 to 2 $\mathrm{cm})$, variations in sampling, post-operative complications (tumor seeding and bleeding) and limited benefit of liver biopsy, the procedure was not performed in this case. ${ }^{4}$

The right adrenal gland was also found to have a welldefined ovoid lesion measuring $3.7 \mathrm{~cm} \times 4.4 \mathrm{~cm} \times 6.4 \mathrm{~cm}$ (Figure 2B). Findings suggestive of metastatic disease of the adrenal gland include a size more than $4 \mathrm{~cm}$ (having $80 \%$ sensitivity and $60 \%$ specificity using a $4 \mathrm{~cm}$ cut-off) or a CT density of more than 20 Hounsfield units. ${ }^{4}$ The patient's abdominal CT findings were consistent with metastatic disease in conjunction with his clinical progression. Chest CT scan revealed bilateral pulmonary nodules (Figure 3). Skeletal survey, however, was unremarkable.

Slide reviews from prior biopsies of the recurrent temporo-zygomatic mass showed sheets of atypical pericytes with numerous thin-walled, ramifying vessels with some dilations. Connective tissue was very scant (Figure 4). These histologic findings were consistent with hemangiopericytoma. Even without the

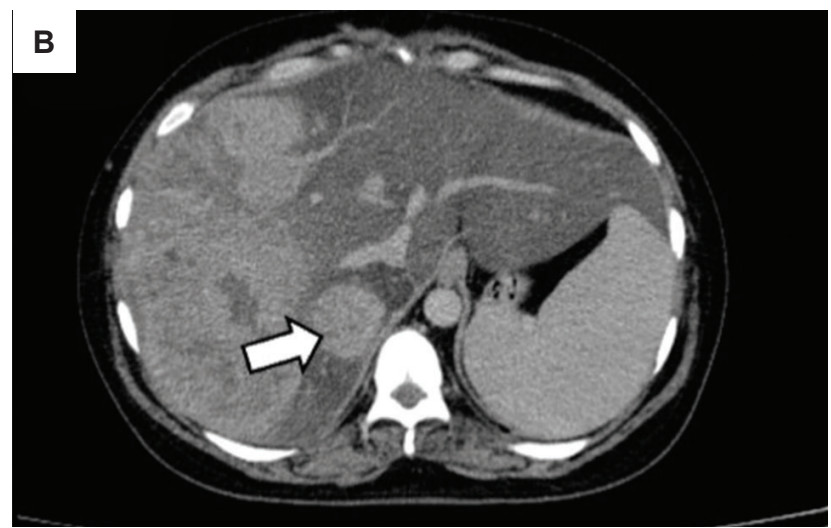

Figure 2. Abdominal computerized tomography scan on axial view showing multiple hepatic (A) and right adrenal metastases (B).
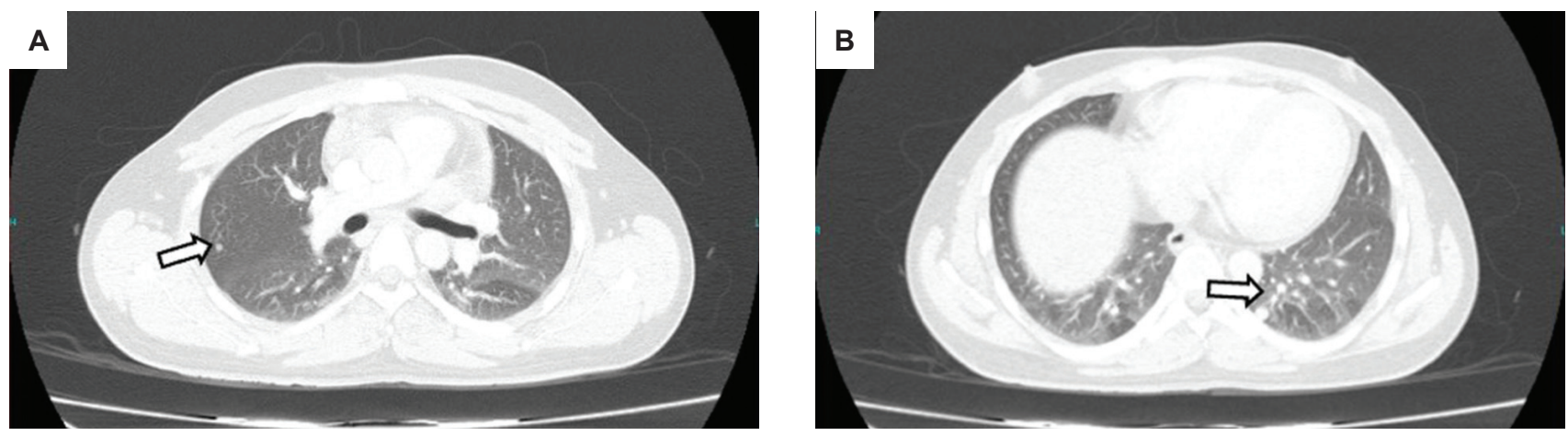

Figure 3. Chest computerized tomography scan in axial view showing bilateral sub-centimeter nodular densities in the apical segment of the right upper lobe (A) and postero-basal segment of the left lower lobe (B). 


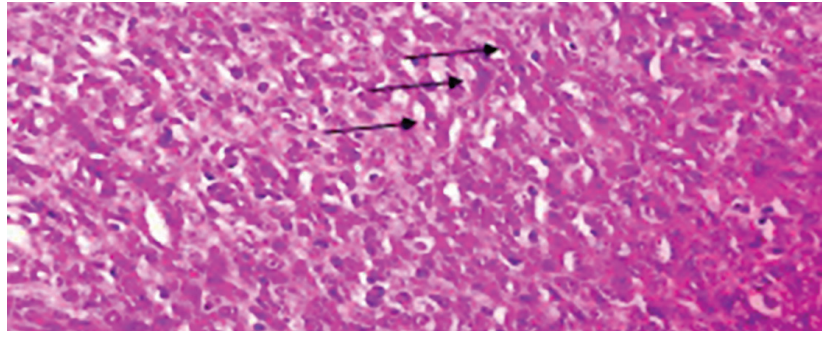

Figure 4. Histopathologic examination of the excised temporo-zygomatic mass. There are pericytes (black arrows) that are round to ovoid, angulated, with hyperchromatic nuclei and basophilic cytoplasm (H\&E, 100x).

ideal benefit of IGF-II, these findings consolidated the presumptive diagnosis of hypoglycemia secondary to hemangiopericytoma or solitary fibrous tumor. ${ }^{1}$ This is a pattern seen in a rare disease entity called Doege-Potter Syndrome.

To prevent recurrence of hypoglycemia, a continuous infusion of $\mathrm{D}_{5} \mathrm{LR} 1 \mathrm{~L}$ with $50 \mathrm{cc} \mathrm{D}_{50} \mathrm{~W}$ was given with close monitoring of CBG levels every six hours. Prednisone was also started at $30 \mathrm{mg}$ once a day. After initiation of steroids, there was a significant improvement of CBG levels, with readings ranging from 90 to $125 \mathrm{mg} / \mathrm{dL}$. A comparison of 48-hour CBG results before and after steroid initiation is shown in Figure 5. The patient was also advised an increase in total caloric intake and

The patient was also referred to Surgery service for evaluation. Due to extensive metastases, he was assessed to be a poor candidate for surgery. He was subsequently referred to Oncology service. Weekly doxorubicin was started with no significant response. He was maintained on steroids, initially for one to three months (or indefinitely depending on treatment response) and inpatient glucose infusion for symptomatic states to prevent recurrent hypoglycemic episodes. Weekly outpatient monitoring of his immune system and adrenal imbalance were done after initiation of steroid treatment.

\section{DISCUSSION}

This case report presents the complexity and rarity of Doege-Potter Syndrome. Various causes of hypoglycemia, including medications such as insulin, alcohol, sepsis and cortisol deficiency, were considered in the process of arriving at a definite diagnosis. ${ }^{4}$ The first three were easily ruled out because the patient had no history of insulin or alcohol use and had no established source of infection. Cortisol deficiency due to right adrenal metastasis was also considered. For adrenal metastases to cause cortisol deficiency, involvement should be bilateral with at least $90 \%$ gland destruction. ${ }^{5}$ These findings were not present in our patient.

Despite its rarity, endogenous hyperinsulinism became a differential diagnosis. The prototypical cause is an insulinoma, an insulin-secreting pancreatic islet B-cell tumor. ${ }^{4}$ This was ruled out after a 72-hour fasting protocol showed decreased post-fasting glucose, insulin and C-peptide levels, in contrast to findings seen in insulinoma (Table 1$)^{6}$

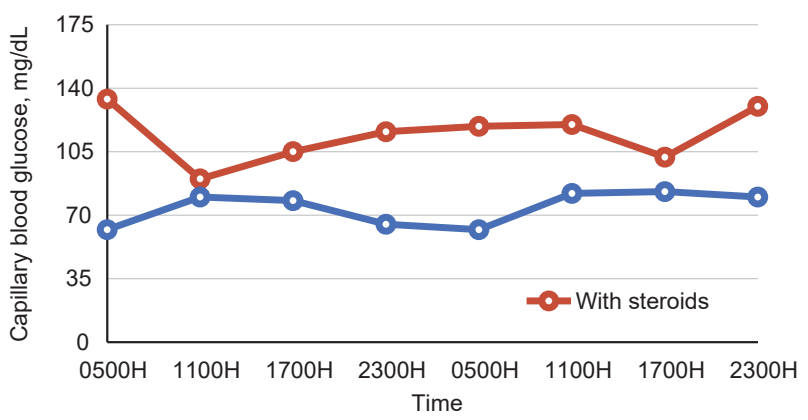

Figure 5. Comparison of 48-hour capillary blood glucose readings with and without Prednisone.

The results of the workup and the patient's temporozygomatic mass with liver and right adrenal gland metastases prompted us to consider non-islet cell tumor hypoglycemia. (NICTH). These solid tumors of mesenchymal and epithelial cell origin may cause hypoglycemia. ${ }^{7,8}$ This was confirmed by the final biopsy report of the excised mass two years prior to the onset of hypoglycemia. It was signed out as a hemangiopericytoma, a non-islet cell tumor.

HPCs are tumors that arise from pericytes, the contractile cells surrounding capillaries and venules. ${ }^{9-11}$ The term solitary fibrous tumor is interchangeable with HPC as these have shared clinical, morphologic, immunophenotypic and genetic features. ${ }^{12}$ Most SFTs are benign neoplasms with $12-13 \%$ rate of malignancy. ${ }^{13}$ Of these malignant SFTs, 10 to $15 \%$ will recur and/or metastasize. ${ }^{14-16} \mathrm{~A}$ case series reported the recurrence rate after a primary resection can be as high as $34 \% .{ }^{17}$ Once with metastases, it may cause significant symptoms such as hypoglycemia, as observed in our patient. Histologic examination of SFTs reveal contractile spindle-shaped pericytes with elongated cell processes around capillaries and postcapillary venules. These, however, may pose difficulties and diagnostic disparities due to its variable fibrous nature, protean cellularity and "pattern-less" pattern. ${ }^{16,18,19}$ SFTs may present with symptoms and signs related to extrinsic compression of adjacent organs. In less than 5\% of cases, it may lead to reactive hypoglycemia, a condition called Doege-Potter syndrome. ${ }^{20-22}$

Doege-Potter syndrome is a rare paraneoplastic syndrome presenting as symptomatic hypoglycemia associated with a solitary fibrous tumor. ${ }^{22,23}$ The SFT ectopically secretes the prohormone of insulin-like growth factor II (IGF-II). ${ }^{11}$ This prohormone suppresses growth hormone and causes decreased synthesis of IGF-binding proteins. ${ }^{24}$ This permits increased levels of unbound and active IGF-II. When the active complexes or unbound IGF-II bind to insulin and IGF receptors in the liver and peripheral tissues, there is a subsequent decrease in the release of glucose into the circulation and increased peripheral glucose metabolism, resulting in hypoglycemia. ${ }^{25}$

The diagnosis requires the determination of serum IGF-I and IGF-II levels; however, these ancillary tests were not available in our country during the course of work-up of our patient. A ratio of IGF-II:IGF-I more than 10 is diagnostic of DPS.7 Our patient's medical history, presentation, histologic and diagnostic findings of metastatic hemangio- 
pericytoma and associated clinical course are sufficient for the presumptive diagnosis of Doege-Potter syndrome. There are 34 compiled cases of DPS from 1930 to $2012 .^{2}$

The management of DPS is multi-dimensional. It includes immediate correction of hypoglycemia, reduction of the tumor burden and prevention of recurrent hypoglycemia if the tumor cannot be controlled. Some case reports have documented that surgical removal of the tumor leads to immediate resolution of hypoglycemia. ${ }^{1}$ However, the presence of widely disseminated metastases made our patient a poor candidate for surgery. Conservatively, the initial and immediate management of hypoglycemia is through the administration of oral and/or intravenous glucose or dextrose-containing fluids when needed. In most cases, this would suffice to avoid further hypoglycemia.,26 Another non-invasive medical approach is to increase the volume and frequency of food intake. ${ }^{27,28}$ Our patient was advised to have small, frequent feedings every 3 hours to prevent the recurrence of hypoglycemia.

Prednisone was also started at $30 \mathrm{mg}$ once daily which alleviated the hypoglycemia. Glucocorticoids such as prednisone, typically in doses of 30 to $60 \mathrm{mg} /$ day, have been most efficient for long-term remission of hypoglycemia. They provide an immediate beneficial effect by suppression of IGF-II and biochemical stimulation of gluconeogenesis and glycogenolysis. ${ }^{29,30}$ As such, glucocorticoids can be taken indefinitely with regular (monthly) evaluation of treatment response and monitoring of side effects such as exogenous Cushing's state or adrenal insufficiency.

Neoadjuvant therapy including chemoradiation and selective embolization of tumoral feeding vessels were also reported as effective. However, these are only limited to patients with resectable masses. Since SFTs are relatively chemotherapy-resistant, there are no standard chemotherapeutic regimens in use at this time, and resection remains the treatment of choice. ${ }^{31}$ In the setting of advanced disease where surgery could not be done or tolerated, systemic therapy may be considered an option. ${ }^{30}$ Our patient received weekly anthracycline chemotherapy with initial improvement in glycemia. Subsequently, he deteriorated after two cycles of treatment.

Promising newer chemotherapeutic agents have been reported. A recent case series of 16 patients with unresectable extracranial metastatic hemangiopericytoma showed partial tumor response in $11(79 \%)$ patients after the administration of temozolomide and bevacizumab. Patients under this empirically designed regimen had a median progression-free and overall survival of 9.7 and 24.3 months, respectively. ${ }^{32}$ Pazopanib, a tyrosine kinase inhibitor that acts on vascular endothelial growth factor and platelet-derived growth factor receptors, has shown favorable effects on survival and response rates for both typical and dedifferentiated/malignant SFTs. ${ }^{33-35}$ These new agents are not yet available in our setting.

\section{CONCLUSION}

Because surgical intervention was not a viable option, conservative medical management of the presumptive Doege-Potter syndrome from a metastatic hemangiopericytoma was challenging. Early screening for possible metastases is important in the prevention of complex sequelae that have the potential to gravely affect the patient's quality of life. We were able to draw several key learning points from our patient's case.

First, a stepwise management approach to DPS should be advocated, including essential ancillary tests, such as the measurement of IGF-I and IGF-II. Second, more aggressive and multimodal therapy may be utilized, such as trans-arterial chemoembolization, metastasectomy or trial of newer chemotherapeutic agents. Lastly, long-term follow-up and close monitoring should be emphasized to both patients and health care providers to mitigate the dismal prognosis of DPS.

\section{Ethical Consideration}

Patient consent was obtained before submission of the manuscript.

\section{Statement of Authorship}

All authors certified fulfillment of ICMJE authorship criteria.

\section{Author Disclosure}

The authors declared no conflict of interest.

\section{Funding Source}

None.

\section{References}

1. Kim DW, Na KJ, Yun JS, Song SY. Doege-Potter syndrome: A report of a histologically benign but clinically malignant case. J Cardiothorac Surg. 2017;12(1):64. PMID: 28784156. PMCID: PMC5547527. https:// doi.org/10.1186/s13019-017-0630-4.

2. Schutt RC, Gordon TA, Bhabhra R, et al. Doege-Potter Syndrome presenting with hypoinsulinemic hypoglycemia in a patient with malignant extrapleural solitary fibrous tumor: A case report. J Med Case Rep. 2013;7:11. PMID: 23302323 PMCID: PMC3546898. https:// doi.org/10.1186/1752-1947-7-11.

3. Bi X, Zhai J, Chun CD. Solitary fibrous tumor of the abdominal wall resurfacing as unilateral pleural effusion and mass: A case report and review of the literature. Respir Med Case Rep. 2018;23:4-7. PMID: 29159031. PMCID: PMC5683803. https://doi.org/10.1016/j. rmcr.2017.10.013.

4. Jameson JL, Kasper DL, Longo DL, Fauci AS, Hauser SL, Loscalzo J, eds. Harrison's Principles of Internal Medicine. 20th ed. Columbus, Ohio: McGraw-Hill Education, 2018.

5. Faulhaber GAM, Borges FK, Ascoli AM, Seligman R, Furlanetto TW. Adrenal failure due to adrenal metastasis of lung cancer: A case report. Case Rep Oncol Med. 2011;2011:326815. PMID: 22606443. PMCID: PMC3350132. https://doi.org/10.1155/2011/326815.

6. Cryer PE, Axelrod L, Grossman AB, et al. Evaluation and management of adult hypoglycemic disorders: An Endocrine Society clinical practice guideline. J Clin Endocrinol Metab. 2009;94(3):709-28. PMID: 19088155. https://doi.org/10.1210/jc.2008-1410.

7. Dutta P, Aggarwal A, Gogate Y, et al. Non-islet cell tumor-induced hypoglycemia: A report of five cases and brief review of the literature. Endocrinol Diabetes Metab Case Rep. 2013;2013:130046. PMID: 24616774. PMCID: PMC3922193. https://doi.org/10.1530/EDM-13-0046.

8. Daughaday WH, Kapadia M. Significance of abnormal serum binding of insulin-like growth factor II in the development of hypoglycemia in patients with non-islet cell tumors. Proc Natl Acad Sci USA. 1989;86(17):6778-82. PMID: 2771956. PMCID: PMC297929. https://doi org/10.1073/pnas.86.17.6778.

9. Stout AP, Murray MR. Hemangiopericytoma: A vascular tumor featuring Zimmermann's pericytes. Ann Surg. 1942;116(1):26-33. PMID: 17858068. PMCID: PMC1543753. https://doi.org/10.1097/00000658194207000-00004.

10. Koch M, Nielsen GP, Yoon SS. Malignant tumors of blood vessels: Angiosarcomas, hemangioendotheliomas, and hemangioperictyomas. J Surg Oncol. 2008;97(4):321-9. PMID: 18286475. https://doi.org/ 10.1002/jso.20973.

11. Hendrick L, Angel W, Besh S, O'Brien T, Goins N, Dickson P. Multidisciplinary management of metastatic hemangiopericytoma presenting with hypoglycemia. Ann Hematol Oncol. 2017; 4(3):1140.

12. Penel N, Amela EY, Decanter G, Robin YM, Marec-Berard P. Solitary fibrous tumors and so-called hemangiopericytoma. Sarcoma. 2012;2012:690251. PMID: 22566753. PMCID: PMC3337510. https:// doi.org/10.1155/2012/690251. 
13. Robinson LA. Solitary fibrous tumor of the pleura. Cancer Control. 2006;13(4):264-9. PMID: 17075563. https://doi.org/10.1177/ 107327480601300403 .

14. Vallat-Decouvelaere AV, Dry SM, Fletcher CD. Atypical and malignant solitary fibrous tumors in extrathoracic locations: Evidence of their comparability to intra-thoracic tumors. Am J Surg Pathol. 1998;22(12):1501-11. PMID: 9850176. https://doi.org/10.1097/ 00000478-199812000-00007.

15. Espat NJ, Lewis JJ, Leung D, et al. Conventional hemangiopericytoma: Modern analysis of outcome. Cancer. 2002;95(8):1746-51. PMID: 12365023. https://doi.org/10.1002/cncr.10867.

16. Ng VY, Scharschmidt TJ, Mayerson JL. Solitary fibrous tumor. 2019. https://emedicine.medscape.com/article/1255879-overview\#a4.

17. Chen LF, Yang Y, Yu XG, Gui QP, Xu BN, Zhou DB. Multimodal treatment and management strategies for intracranial hemangiopericytoma. J Clin Neurosci. 2015;22(4):718-25. PMID: 25744076. https://doi.org/10.1016/j.jocn.2014.11.011.

18. Wu R. Solitary fibrous tumor. PathologyOutlines.com website. https:// www.pathologyoutlines.com/topic/lungtumorsolitaryfibroustumor. html. Accessed 27 February 2021.

19. Geramizadeh B, Marzban M, Churg A. Role of immunohistochemistry in the diagnosis of solitary fibrous tumor, a review. Iran J Pathol. 2016;11(3):195-203. PMID: 27799967. PMCID: PMC5079451.

20. England DM, Hochholzer L, McCarthy MJ. Localized benign and malignant fibrous tumors of the pleura. A clinicopathologic review of 223 cases. Am J Surg Pathol. 1989;13(8):640-58. PMID: 2665534 https://doi.org/10.1097/00000478-198908000-00003.

21. Kalebi AY, Hale MJ, Wong ML, Hoffman T, Murray J. Surgically cured hypoglycemia secondary to pleural solitary fibrous tumour: Case report and update review on the Doege-Potter syndrome. Cardiothorac Surg. 2009;4:45. PMID: 19689813. PMCID: PMC2739517. https://doi.org/10.1186/1749-8090-4-45.

22. Roy TM, Burns MV, Overly DJ, Curd BT. Solitary fibrous tumor of the pleura with hypoglycemia: The Doege-Potter syndrome. J Ky Med Assoc. 1992;90(11): 557-60. PMID:1474302.

23. Chamberlain MH, Taggart DP. Solitary fibrous tumor associated with hypoglycemia: An example of the Doege-Potter syndrome. Thorac Cardiovasc Surg. 2000;119(1):185-7. PMID: 10612786. https:// doi.org/10.1016/s0022-5223(00)70242-x.

24. Davda R, Seddon BM. Mechanisms and management of non-islet cell tumour hypoglycaemia in gastrointestinal stromal tumour: Case report and a review of published studies. Clin Oncol ( $\mathrm{R}$ Coll Radiol). 2007;19(4):265-8. PMID: 17433971. https://doi.org/10.1016/ j.clon.2006.12.008
25. Sohda T, Yun K. Insulin-like growth factor II expression in primary meningeal hemangiopericytoma and its metastasis to the liver accompanied by hypoglycemia. Hum Pathol. 1996;27(8):858-61. PMID: 8760024. https://doi.org/10.1016/s0046-8177(96)90463-3.

26. Strâmbu IR, Leonte DG, Bolca CN. Large pleural tumor revealed by severe hypoglycemia: Doege-Potter syndrome. Rom J Morphol Embryol. 2017;58(1):287-91. PMID: 28523334.

27. Holt RI, Teale JD, Jones JS, Quin JD, McGregor AM, Miell JP. Gene expression and serum levels of insulin-like growth factors (IGFs) and IGF-binding proteins in a case of non-islet cell tumour hypoglycaemia. Growth Horm IGF Res. 1998;8(6):447-54. PMID: 10985756. https:// doi.org/10.1016/s1096-6374(98)80297-9.

28. Trivedi N, Mithal A, Sharma AK, et al. Non-islet cell tumour induced hypoglycaemia with acromegaloid facial and acral swelling. Clin Endocrinol (Oxf). 1995;42(4):433-5. PMID: 7750199. https://doi org/10.1111/j.1365-2265.1995.tb02654.x.

29. Teale JD, Wark G. The effectiveness of different treatment option for non-islet cell tumour hypoglycaemia. Clin Endocrinol (Oxf) 2004;60(4):457-60. PMID: 15049960. https://doi.org/10.1111/j.13652265.2004.01989x

30. Bodnar TW, Acevedo MJ, Pietropaolo M. Management of non-isletcell tumor hypoglycemia: A clinical review. J Clin Endocrinol Metab. 2014;99(3):713-22. PMID: 24423303. PMCID: PMC5393479. https:// doi.org/10.1210/jc.2013-3382.

31. Han G, Zhang Z, Shen $X$, et al. Doege-Potter syndrome: A review of the literature including a new case report. Medicine (Baltimore) 2017;96(27):e7417. PMID: 28682900. PMCID: PMC5502173. https:// doi.org/10.1097/MD.0000000000007417.

32. Park MS, Patel SR, Ludwig JA, et al. Activity of temozolomide and bevacizumab in the treatment of locally advanced, recurrent, and metastatic hemangiopericytoma and malignant solitary fibrous tumor. 2011;117(21):4939-47. PMID: 21480200. PMCID: PMC3135685. NIHMSID: NIHMS277775. https://doi.org/10.1002/cncr.26098.

33. Lee ATJ, Benson C. Pazopanib as a choice for advanced solitary fibrous tumour. Lancet Oncol. 2020;21(3):332-3. PMID: 32066545. https:// doi.org/10.1016/S1470-2045(20)30018-8.

34. Martin-Broto J, Cruz J, Penel N, et al. Pazopanib for treatment of typical solitary fibrous tumours: A multicentre, single-arm, phase 2 trial. Lancet Oncol. 2020;21(3):456-66. PMID: 32066540. https:// doi.org/10.1016/S1470-2045(19)30826-5.

35. Martin-Broto J, Stacchiotti S, Lopez-Pousa A, et.al. Pazopanib for treatment of advanced malignant and dedifferentiated solitary fibrous tumour: A multicentre, single-arm, phase 2 trial. Lancet Oncol. 2019;20(1):134-44. PMID: 30578023. https://doi.org/10.1016/S14702045(18)30676-4.

\section{ARTICLE COMMENTARY}

Evaluating a case report for Doege-Potter Syndrome without the confirmatory diagnostic tests, in this case a ratio of IGFII:IGF-I > 10, is at best a presumptive diagnosis from a clinical association between a solid fibrous tumor and persistent in-hospital hypoglycemia. Hence, due to the lack of the confirmatory test results there was initially some hesitation to publish this paper. Nevertheless, because of the rarity of the condition and to maximize every opportunity for learning, JAFES decided to publish this case report to highlight the difficulty and limitations of endocrine practice in South East and South Asia. The challenges include the limited availability of tests in some ASEAN member countries; the prohibitive costs to the patient if the tests are sent out of the country; and the consequent delay in diagnosis.

By doing so, it is hoped that we can stimulate a discussion that will provoke member Societies to 1) Advocate and raise funds for a complete endocrine testing facility in a government or private hospital in their capital cities or regional hubs; 2) Set aside funds for this specific purpose to which endocrine training institutions can apply for financial assistance; and 3) Establish a laboratory network within the ASEAN to facilitate diagnosis of rare and interesting cases or diagnostic dilemmas that can eventually be published.

\section{Patricia B. Gatbonton, MD, FPCP, FPSEDM}

Past President, Philippine Society of Endocrinology, Diabetes and Metabolism

Authors are required to accomplish, sign and submit scanned copies of the JAFES Author Form consisting of: (1) Authorship Certification, that authors contributed substantially to the work, that the manuscript has been read and approved by all authors, and that the requirements for authorship have been met by each author; (2) the Author Declaration, that the article represents original material that is not being considered for publication or has not been published or accepted for publication elsewhere, that the article does not infringe or violate any copyrights or intellectual property rights, and that no references have been made to predatory/suspected predatory journals; (3) the Author Contribution Disclosure, which lists the specific contributions of authors; (4) the Author Publishing Agreement which retains author copyright, grants publishing and distribution rights to JAFES, and allows JAFES to apply and enforce an Attribution-Non-Commercial Creative Commons user license; and (5) the Conversion to Visual Abstracts ("optional for original articles only) to improve dissemination to practitioners and lay readers Authors are also required to accomplish, sign, and submit the signed ICMJE form for Disclosure of Potential Conflicts of Interest. For original articles, authors are required to submit a scanned copy of the Ethics Review Approval of their research as well as registration in trial registries as appropriate. For manuscripts reporting data from studies involving animals, authors are required to submit a scanned copy of the Institutional Animal Care and Use Committee approval. For Case Reports or Series, and Images in Endocrinology, consent forms, are required for the publication of information about patients; otherwise, appropriate ethical clearance has been obtained from the institutional review board. Articles and any other material published in the JAFES represent the work of the author(s) and should not be construed to reflect the opinions of the Editors or the Publisher. 